\title{
Plasma miR-136 can be used to screen patients with knee osteoarthritis from healthy controls by targeting IL-17
}

\author{
LEI WAN ${ }^{1}$, QIANG ZHAO ${ }^{2}$, GANG NIU $^{3}$, TAI XIANG ${ }^{4}$, CUILEI DING $^{4}$ and SHIJIE WANG ${ }^{5}$ \\ ${ }^{1}$ Department of Joint Surgery, Rizhao People's Hospital; ${ }^{2}$ Department of Bone Surgery, The Second People's Hospital of \\ Rizhao City; ${ }^{3}$ Surgical Department, Rizhao People's Hospital; ${ }^{4}$ Department of Surgery, Rizhao People's Hospital; \\ ${ }^{5}$ Department of Orthopedics, Rizhao People's Hospital, Rizhao, Shandong 276800, P.R. China
}

Received February 5, 2018; Accepted August 7, 2018

DOI: $10.3892 /$ etm.2018.6625

\begin{abstract}
The current study mainly evaluated the plasma level of microRNA (miR)-136 in knee osteoarthritis (KOA) patients and determined if miR-136 could be used as a potential biomarker to screen KOA patients from healthy controls. It was demonstrated that plasma miR-136 was significantly decreased in the plasma of KOA patients. Moreover, the reduction in plasma miR-136 negatively correlated with the severity of KOA. Additionally, the increase in the serum interleukin (IL)-17 level positively correlated with the severity of KOA. More importantly, dual luciferase assays and western blot assays indicated that IL-17 was a target gene of miR-136. Further analysis showed that plasma miR-136 could be used as a biomarker to screen KOA patients from healthy controls. In summary, for the first time, the present study revealed that through targeting IL-17, decreased plasma miR-136 levels could be used as a potential biomarker to screen KOA patients from healthy controls.
\end{abstract}

\section{Introduction}

Knee osteoarthritis (KOA) is a common disease in the elderly population (1). Due to the nonrenewable nature of the articular cartilage, once KOA exists, the quality of life in elderly patients is largely affected (1-3). According to a statistical analysis, the incidence of KOA in China has reached 3-15.6\%, and the annual cost of treatment for KOA is a heavy burden for the family and society (4). Currently, early treatment of KOA mainly depends on conservative drug treatments. At the advanced stage, patients usually undergo artificial total knee arthroplasty $(5,6)$. However, there are a many controversies regarding surgery for patients younger than 60 years of age since surgical trauma will bring many disadvantages.

Correspondence to: Dr Shijie Wang, Department of Orthopedics, Rizhao People's Hospital, 126 Taian Road, Donggang, Rizhao, Shandong 276800, P.R. China

E-mail: ag201801888@163.com

Key words: miR-136, knee osteoarthritis, interleukin-17, biomarker
Moreover, postoperative rehabilitation treatment requires a long time to ensure the recovery of knee joint function in patients. Hence, early detection and prevention of KOA are of great importance for high-risk patients.

Interleukin (IL-17) is a novel cytokine family secreted mainly by $\mathrm{CD}^{+}{ }^{+} \mathrm{T}$ helper 17 cells (Th17), including IL-17A, IL-17B, IL-17C, IL-17D, IL-17E and IL-17F (7,8). By binding the corresponding receptors on the cell surface, IL-17 is widely involved in bone metabolism $(9,10)$. Multiple studies have confirmed that IL-17 is the main factor leading to osteoarthritis bone injury (9-11). Clinical data also indicate that the levels of IL-17 and IL-17R are higher in the synovial fluid of patients with arthritis (11). Furthermore, high levels of IL-17 and TNFa are correlated with poor prognosis for osteoarthritis patients $(12,13)$. However, the underlying mechanism by which IL17 is highly upregulated in osteoarthritis has been poorly understood.

MicroRNAs (miRNAs/miRs) are small noncoding RNAs with approximately 23 nucleotides that extensively participate in posttranscriptional regulation by binding the 3 ' untranslated region (3'UTR) of mRNA $(14,15)$. Due to the ability to be resistant to RNase, increasing evidence has suggested the use of circulating miRNAs as potential biomarkers for various diseases (16). For instance, increased levels of miR-29c, miR-93, and miR-126 are shown to be related to the progression of osteoarthritis (16).

Abnormal expression of miR-136 has been widely reported in the development of tumors and metabolic diseases $(17,18)$. However, we still lack knowledge regarding whether miR-136 is involved in the progression of KOA. The current study explored the plasma level of miR-136 in KOA patients and attempted to determine if it could be used as a potential biomarker to screen KOA patients from healthy controls.

\section{Materials and methods}

Patient samples. This study was approved by the Ethics Committee of Rizhao People's Hospital (Rizhao, China) and performed in accordance with the Declaration of Helsinki. All participants gave their informed consent.

In total, 50 patients with knee OA were recruited from February 2016 to December 2016 at the Department of Joint Surgery, Rizhao People's Hospital, according to the 1986 classification of osteoarthritis of the knee in the diagnostic criteria 
of the American Rheumatism Association (19). Diagnosis of osteoarthritis was performed according to criteria of the American College of Rheumatology (19). Exclusion criteria were: i) Symptoms suggestive of any other chronic inflammatory disease; ii) diabetes; iii) history of corticosteroid treatment; iv) any other form of arthritis; v) cancer; and vi) family history of osteoarthritis. X-ray examinations were carried out to divide patients into early, middle and late KOA stages. All participants were examined with bilateral knee plain radiographs (bilateral anteroposterior and lateral) using an SD 3000 Synchro Stand. Two independent radiologists evaluated radiographic changes related to $\mathrm{OA}$ using the $\mathrm{K} / \mathrm{L}$ grading system (20). KOA was defined as a $\mathrm{K} / \mathrm{L}$ grade $\geq 2$ in both knees. In addition, health volunteers who were 35 years of age and gender matched at the Department of Physical Examination were enrolled in the study as health controls (HCs). The details of KOA patients and healthy controls are listed in Table I.

Sample acquisition and RNA extraction. A $5 \mathrm{ml}$ aliquot of blood was collected from all participants and directly into sodium citrate tubes. Total RNA was isolated with RNAVzol LS (Vigorous, Beijing, China) according to the manufacturer's instructions for isolating small RNAs. The quality, quantity and integrity of RNA were monitored using a NanoDrop spectrophotometer (ND-1000; NanoDrop Technologies; Thermo Fisher Scientific, Inc., Wilmington, DE, USA).

$q P C R$ validation. RNA was reverse transcribed into cDNA using a PrimeScript OneStep RT-qPCR kit (C28025-032; Invitrogen; Thermo Fisher Scientific, Inc., Waltham, MA, USA). The detailed RT-qPCR procedure is described as follows: $95^{\circ} \mathrm{C}$ for $10 \mathrm{~min}$; followed by 50 cycles of $95^{\circ} \mathrm{C}$ for $10 \mathrm{sec}, 55^{\circ} \mathrm{C}$ for $10 \mathrm{sec}, 72^{\circ} \mathrm{C}$ for $5 \mathrm{sec}, 99^{\circ} \mathrm{C}$ for $1 \mathrm{sec}, 59^{\circ} \mathrm{C}$ for $15 \mathrm{sec}$, and $95^{\circ} \mathrm{C}$ for $1 \mathrm{sec}$; and then cooled to $40^{\circ} \mathrm{C}$. The relative expression levels were calculated with the $2^{-\Delta \Delta C q}$ method (21), and the experiments were repeated in triplicate. The specific primers used in the current study are listed as follows: miR-136-RT: GTCGTATCCAGTGCAGGGTCC GAGGTATTCGCACTGGATACGACAACAC; U6-RT: GTCGTATCCAGTGCAGGGTCCGAGGTATTCGCACTG GATACGACAAATATG; miR-136-F: GCGCTGGAGTGT GACAATGGTG; U6-F: GCGCGTCGTGAAGCGTTC; Universal reverse primer: GTGCAGGGTCCGAGGT.

Enzyme-linked immunosorbent assay (ELISA). The serum samples were used to quantify the level of IL-17 using an enzyme-linked immunosorbent assay according to the manufacturer's instructions (R\&D Systems, Inc., Minneapolis, MN, USA). Samples were read at a $450 \mathrm{~nm}$ wavelength using a microplate reader (Model 3550; Thermo Fisher Scientific, Inc.).

Cell culture. 293 cells were seeded at a density of $1.5 \times 10^{4}$ cells $/ \mathrm{cm}^{2}$ and cultured in Dulbecco's modified Eagle's medium (HyClone; GE Healthcare Life Sciences, Logan, UT, USA) containing 10\% heat-inactivated fetal calf serum (Gibco; Thermo Fisher Scientific, Inc.), streptomycin (100 mg/ml, Gibco; Thermo Fisher Scientific, Inc.), and penicillin (100 U/ml; Gibco; Thermo Fisher Scientific, Inc.) in an incubator at $37^{\circ} \mathrm{C}$ with $5 \% \mathrm{CO}_{2}$.
Table I. Comparison of patients baseline data between groups.

\begin{tabular}{lccc}
\hline Characteristic & KOAs $(\mathrm{n}=74)$ & $\mathrm{HCs}(\mathrm{n}=79)$ & P-value \\
\hline Age (mean \pm SE, years) & $65.8 \pm 7.3$ & $66.2 \pm 6.9$ & 0.451 \\
Sex (female/male, $\mathrm{n})$ & $40 / 34$ & $42 / 37$ & 0.298 \\
BMI (mean $\left.\pm \mathrm{SE}, \mathrm{kg} / \mathrm{m}^{2}\right)$ & $26.7 \pm 1.5$ & $26.8 \pm 1.2$ & 0.353 \\
K/L grade $(2 / 3 / 4)$ & $22 / 29 / 23$ & - &
\end{tabular}

BMI, body mass index; KOA, knee osteoarthritis; HC, healthy control; SE, standard error.

miRNA target prediction and dual-luciferase reporter assay. miRNA targets were predicted using TargetScan (https://www.targetscan.org). The 3'UTR of IL-17, which contains the predicted target site for miR-136, was cloned into the pmirGLO luciferase reporter vector (Promega Corporation, Madison, WI, USA), which was cleaved at SacI and XhoI sites. The details of the PCR procedures are described as follows: A hot start step at $95^{\circ} \mathrm{C}$ for $10 \mathrm{~min}$, followed by 40 cycles of $95^{\circ} \mathrm{C}$ for $15 \mathrm{sec}, 55^{\circ} \mathrm{C}$ for $45 \mathrm{sec}$ and $72^{\circ} \mathrm{C}$ for $30 \mathrm{sec}$.

Prior to conducting the dual reporter assay, $5 \times 10^{4}$ 293 cells/well were seeded in 24 -well plates with $500 \mu 1$ DMEM and cultured for $18 \mathrm{~h}$. The cells were transfected with the modified firefly luciferase reporter vector $(500 \mathrm{ng} / \mu \mathrm{l})$ and mixed with Vigofect transfection reagent (Vigorous, Beijing, China) according to the manufacturer's protocol. After continuous exposure to miR-136/pmirGLO-IL-17-3'UTR or $\mathrm{NC} /$ pmirGLO blank vector for $48 \mathrm{~h}$, the firefly and Renilla luciferase activities were measured with the Dual-Luciferase ${ }^{\circledR}$ Reporter Assay system (Promega Corporation) according to the manufacturer's protocol. Firefly luciferase activity was normalized to Renilla luciferase activity.

Cell transfection. miR-136 mimic, inhibitor and negative control (NC) were purchased from Genepharma Co., Ltd. (Shanghai, China). In brief, $6 \times 10^{5}$ cells were equally seeded in 6-well plates with $2 \mathrm{ml}$ DMEM containing serum and antibiotics. At the same time, miR-136 mimic, inhibitor or NC was mixed with HiPerFect transfection reagent (Qiagen, Inc., Valencia, CA, USA) and incubated at room temperature for $10 \mathrm{~min}$. The complex was then transfected into the cells for $48 \mathrm{~h}$.

Western blotting. Cell protein was extracted using radioimmunoprecipitation lysis buffer (Beijing Solarbio Science \& Technology Co., Ltd., Beijing, China) and was collected following centrifugation at $12,000 \mathrm{x}$ g for $30 \mathrm{~min}$ at $4{ }^{\circ} \mathrm{C}$. A bicinchoninic protein assay kit (Pierce; Thermo Fisher Scientific, Inc.) was used to determine the protein concentration. A total of $15 \mu \mathrm{g}$ protein was loaded per lane, separated by $10 \%$ SDS-PAGE and transferred to polyvinylidene difluoride membranes. The membranes were blocked with $8 \%$ non-fat dry milk at $4^{\circ} \mathrm{C}$ overnight. Following three washes with PBS with Tween 20 (5 min/wash), the membranes were incubated with the following primary antibodies at $4^{\circ} \mathrm{C}$ overnight: IL-17 (cat no. 13838; 1:1,000; Cell Signaling Technology, Inc., Danvers, MA, USA) and GAPDH (cat. no. 5174; 1:1,000; 

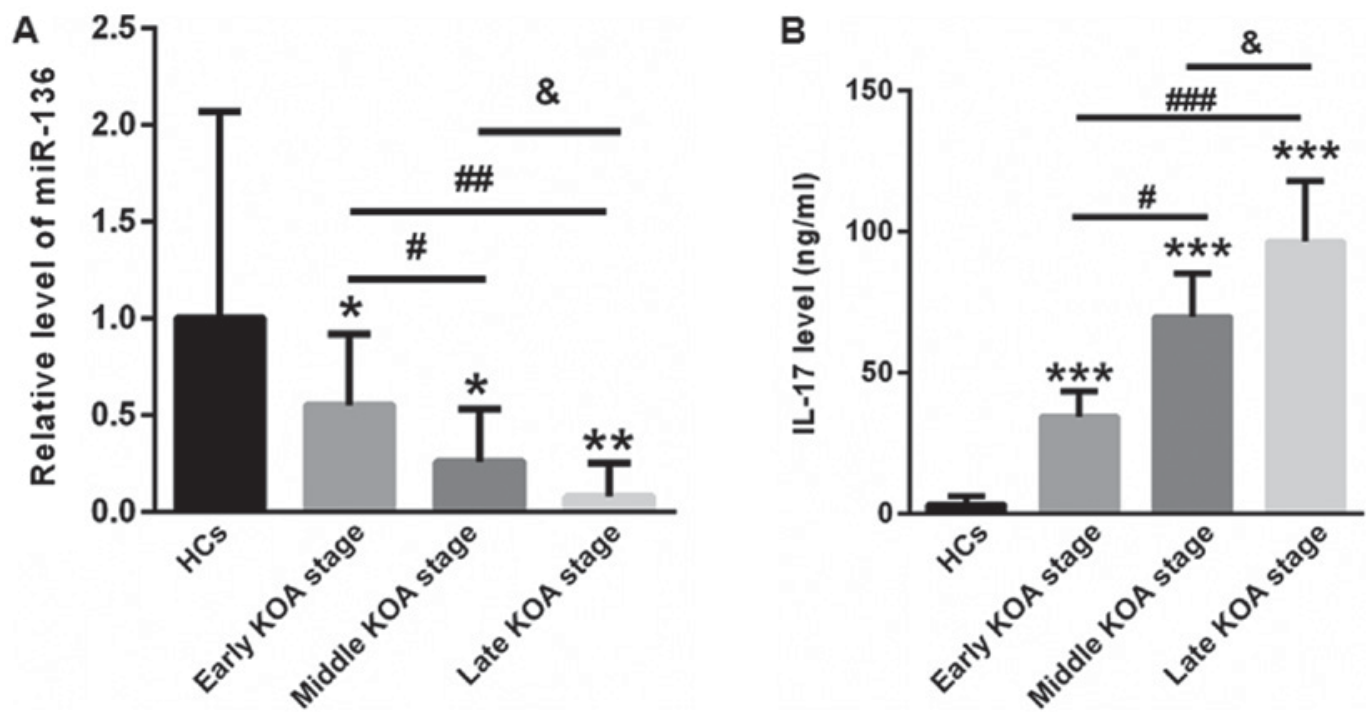

Figure 1. Plasma miR-136 levels were reduced and serum IL-17 levels were increased in KOA patients compared with those in healthy controls. (A) qPCR analysis showed that plasma levels of miR-136 were significantly decreased in patients at the early, middle and late KOA stages, respectively. (B) In contrast to healthy controls, the levels of IL-17 were increased in patients at the early, middle and late KOA stages. ${ }^{*} \mathrm{P}<0.05,{ }^{* *} \mathrm{P}<0.01,{ }^{* * *} \mathrm{P}<0.001$ vs. healthy control; ${ }^{\#} \mathrm{P}<0.05,{ }^{\# /} \mathrm{P}<0.01,{ }^{\# \# \#} \mathrm{P}<0.001$; ${ }^{\circledR} \mathrm{P}<0.05$ vs. as indicated. miR, microRNA; IL, interleukin; KOA, knee osteoarthritis.
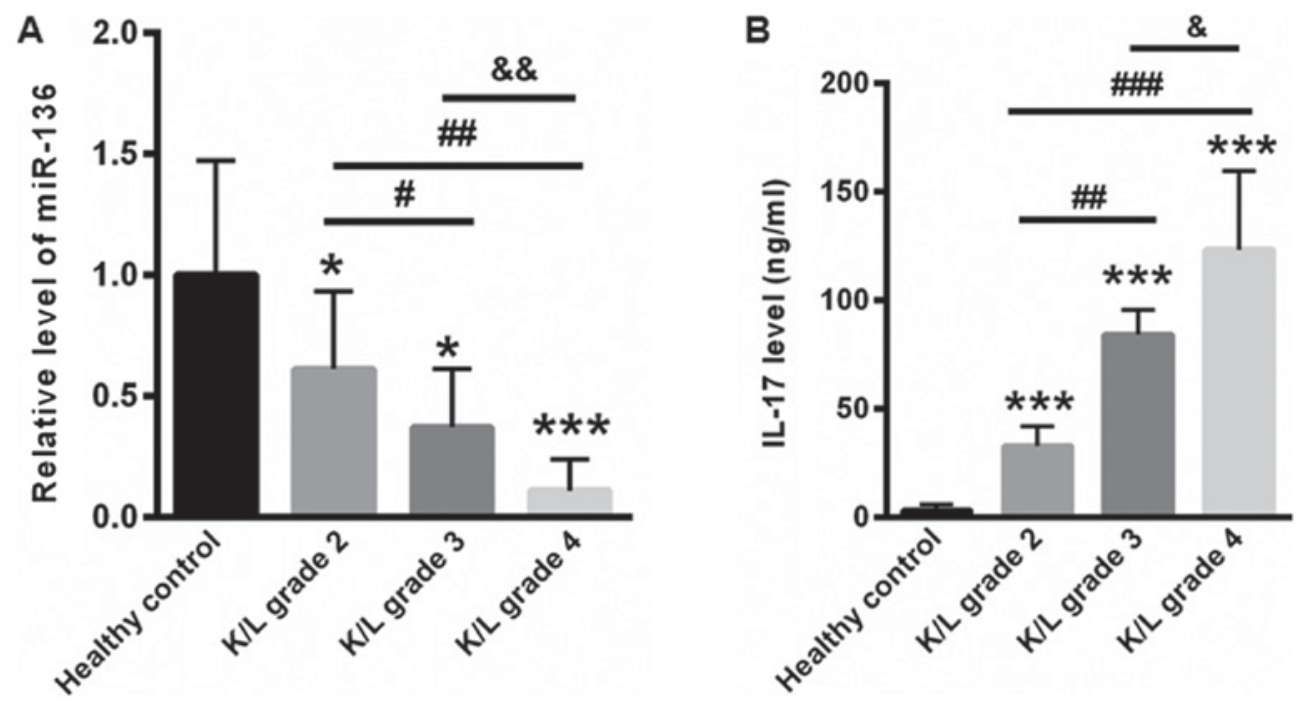

Figure 2. miR-136 was reduced along with increased K/L grades in KOA patients. (A) qPCR analysis showed that plasma miR-136 levels were much lower along with decreased K/L grades in KOA patients. (B) Compared with healthy controls, the levels of IL-17 were significantly increased for K/L grade 2, 3, and 4 KOA patients. ${ }^{*} \mathrm{P}<0.05,{ }^{* * *} \mathrm{P}<0.001$ vs. healthy controls; ${ }^{\# P} \mathrm{P}<0.05,{ }^{\# \#} \mathrm{P}<0.01,{ }^{\# \# t} \mathrm{P}<0.001$; ${ }^{\&} \mathrm{P}<0.05{ }^{\text {\&\&}} \mathrm{P}<0.01$ vs. as indicated. miR, microRNA; IL, interleukin; KOA, knee osteoarthritis.

Cell Signaling Technology, Inc.). Following several washes with TBST, the membranes were incubated with horseradish peroxidase (HRP)-conjugated goat anti-rabbit and anti-mouse Immunoglobulin $\mathrm{G}$ ( $\mathrm{IgG}$ ) or HRP-conjugated mouse anti-goat IgG (all 1:5,000; Zhongshan Gold Bridge Biological Technology Co., Beijing, China) for $2 \mathrm{~h}$ at room temperature and then washed. The blots were then incubated with horseradish peroxidase (HRP)-conjugated anti-IgG secondary antibody (1:5,000; OriGene Technologies, Inc., Beijing, China) for $2 \mathrm{~h}$ at room temperature and then washed, followed by detection with enhanced chemiluminescent substrate (EMD Millipore, Billerica, MA, USA). GAPDH was used as an internal control. ImageJ software (National Institutes of Health, Bethesda, MD, USA) was used for density analysis.
Receiver operating characteristic (ROC) curves. An ROC curve was established to interpret the ability of miR-136 in discriminating KOA patients from healthy controls. In brief, the fluorescence signals of each reaction well were collected quantitatively, and the $\mathrm{Cq}$ values were recorded. The expression of miR-136 in KOA patients relative to healthy control was expressed as $2^{-\Delta \Delta C q} . \Delta \mathrm{Cq}$ is the difference of $\mathrm{Cq}$ values of the target gene to the internal reference U6 in each sample, namely $\Delta \mathrm{Cq}=\mathrm{Cq}(\mathrm{miR}-136)-\mathrm{Cq}(\mathrm{U} 6), \Delta \Delta \mathrm{Cq}=(\mathrm{Cq}$ miR-136-Cq U6) KOA-(Cq miR-136-Cq U6) normal. To perform ROC analysis, $2^{-\Delta \Delta C q}$ was recorded parallel to the diagnostic results of gold standard, that is healthy control $=0, \mathrm{KOA}$ patients $=1$. Then, the area under the curve (AUC), sensitivity and specificity were computed in order to validate the diagnostic application of 
A
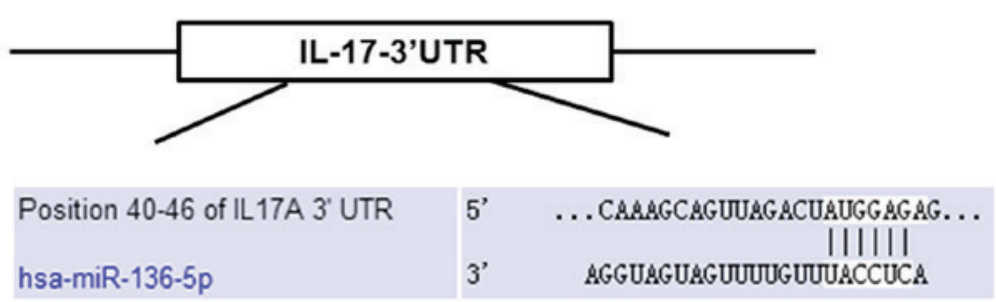

B

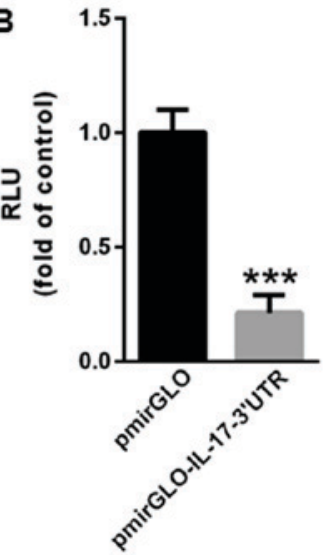

C

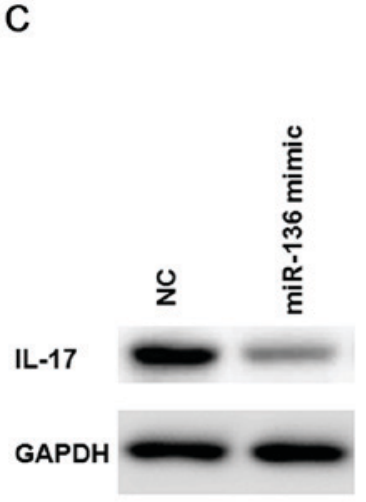

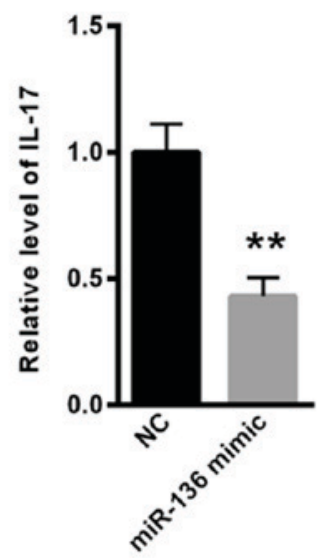
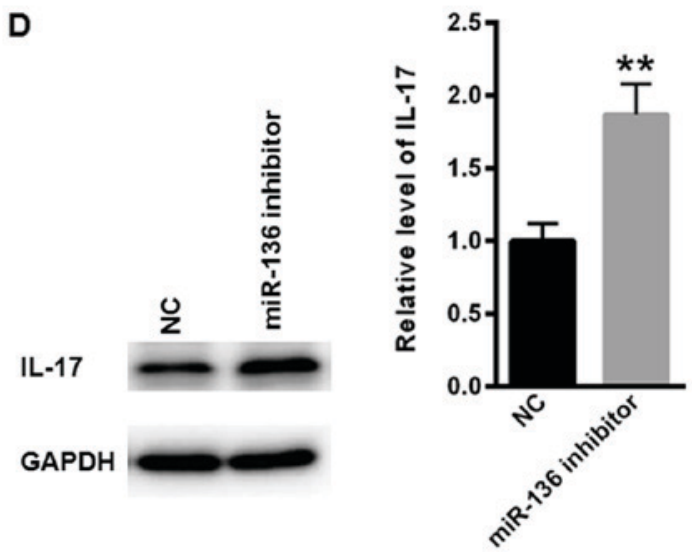

Figure 3. IL-17 is a target gene of miR-136. (A) TargetScan analysis showed a conserved binding site of miR-136 in the 3'UTR of IL-17. (B) Dual-luciferase reporter assay showed that miR-136 significantly suppressed the relative luciferase activity of pmirGLO-IL-17-3'UTR compared with the blank pmirGLO plasmid. Western blot assay also indicated that overexpression of (C) miR-136 significantly suppressed the protein level of IL-17, while inhibition of (D) miR-136 significantly increased the expression of IL-17. ${ }^{* *} \mathrm{P}<0.01,{ }^{* * * *} \mathrm{P}<0.001$ vs. healthy controls. miR, microRNA; IL, interleukin; 3'UTR, 3' untranslated region.

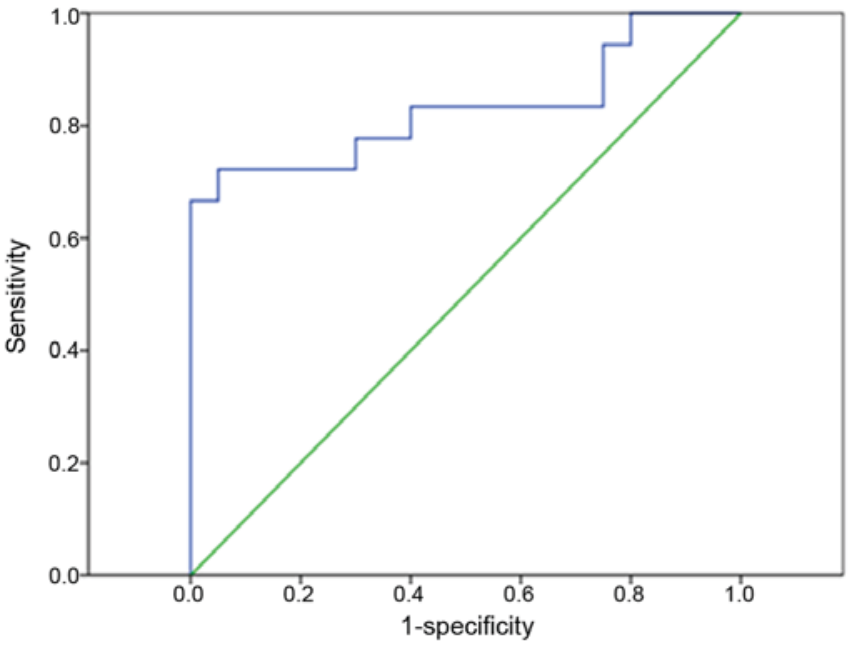

Figure 4. ROC analysis showed that miR-136 could be used as a biomarker to screen KOA patients from healthy controls. miR, microRNA; ROC, Receiver operating characteristic; KOA, knee osteoarthritis.

miR-136 as KOA biomarkers in contrast to the diagnostic results of gold standard (version 20.0, SPSS, Inc., Chicago, Illinois).

Statistics. The data are represented as the mean \pm standard error. The two-tailed unpaired Student's t-tests were used for comparisons of the two groups. For multiple groups comparisons, one-way analysis of variance followed by Tukey's post hoc test was used. Statistical tests were performed using SPSS software (version 13.0; SPSS, Inc., Chicago, IL, USA). P $<0.05$ was considered to indicate a statistically significant difference.

\section{Results}

Reduced plasma miR-136 and increased serum IL-17 levels in KOA patients. First, we examined the plasma levels of miR-136 in KOA patients and healthy controls. Compared with healthy controls $(1 \pm 1.07)$, the plasma levels of miR-136 were significantly increased to $0.55 \pm 0.37,0.26 \pm 0.27$, and $0.08 \pm 0.17$ for patients at the early, middle and late KOA stages, respectively (Fig. 1A). Next, serum IL-17 levels were determined using an ELISA kit. In contrast to healthy controls $(3.23 \pm 2.86 \mathrm{ng} / \mathrm{ml})$, the levels of IL-17 for patients at the early, middle and late KOA stages were $34.37 \pm 8.93,69.65 \pm 15.41$, and $96.42 \pm 21.45 \mathrm{ng} / \mathrm{ml}$, respectively (Fig. 1B).

miR-136 was reduced along with increased K/L grades. Moreover, we examined miR-136 and Il-17 levels based on the $\mathrm{K} / \mathrm{L}$ grade. Our data showed that the level of plasma miR-136 was $1 \pm 0.47$ but was much lower in the K/L grade $2(0.61 \pm 0.32)$, grade $3(0.37 \pm 0.24)$, and grade $4(0.11 \pm 0.13)$ groups (Fig. 2A). In addition, we determined the serum IL-17 levels 
according to the $\mathrm{K} / \mathrm{L}$ grade. Compared with healthy controls $(3.23 \pm 2.86 \mathrm{ng} / \mathrm{ml})$, the levels of IL-17 for $\mathrm{K} / \mathrm{L}$ grade 2, 3, and $4 \mathrm{KOA}$ patients were $32.87 \pm 8.97,84.32 \pm 11.45$, and $123.34 \pm 36.45 \mathrm{ng} / \mathrm{ml}$, respectively (Fig. 2B).

IL-17 is a target gene of miR-136. The above observations motivated us to explore the underlying mechanism by which miR-136 levels were negatively correlated with the serum levels of IL-17. TargetScan analysis showed a conserved binding site of miR-136 in the 3'UTR of IL-17 (Fig. 3A). Dual-luciferase reporter assay showed that miR-136 significantly suppressed the relative luciferase activity of pmirGLO-IL-17-3'UTR, compared with the blank pmirGLO plasmid (Fig. 3B). The western blot assay also indicated that overexpression of miR-136 significantly suppressed the protein level of IL-17 (Fig. 3C), while inhibition of miR-136 significantly increased the expression of IL-17 (Fig. 3D). These data validated that IL-17 was a target gene of miR-136.

miR-136 could be used to screen KOA patients from healthy controls. We then evaluated whether miR-136 could be used as a potential biomarker for patients with KOA. ROC analysis showed that the plasma miR-136 level could be used to screen KOA patients from healthy controls, with an ROC curve area of 0.935 (95\% confidence interval: $0.817-1.000 ; \mathrm{P}<0.0001)$ (Fig. 4).

\section{Discussion}

$\mathrm{KOA}$, a chronic progressive disease, is a common disease in the elderly. With the advent of an aging society, the incidence of this disease is increasing $(22,23)$. Undoubtedly, it is of great significance to improve the quality of life of patients with KOA (5).

Increasing evidence has associated key roles of miRs with the progression of KOA $(24,25)$. For example, miR-9 is shown to modulate the development of KOA via the NF-kappaB1 pathway in chondrocytes (24). miR-29a is also reported to suppress synovitis in the pathogenesis of KOA by targeting VEGF (25). Abnormal expression of miR-136-5p after spinal cord injury has been identified. He et al reported that overexpression of miR-136-5p effectively enhanced inflammatory factors and chemokines via activating NF- $\kappa$ B/A20 signaling in the IL-17-mediated inflammatory response both in vitro and in vivo (26). In IL-17-stimulated astrocytes, miR-136-5p is also found to increase inflammatory responses via suppressing the expression of A20 (27). However, whether miR-136 is involved in IL-17 induced inflammatory responses in KOA patients has never been explored. In the current study, we mainly focused on miR-136, which has been extensively studied in various diseases. However, there have been limited studies on the role of miR-136 in the pathology of KOA. Here, we showed novel data that the plasma miR-136 level was significantly decreased in the plasma of KOA patients. Moreover, the reduction in plasma miR-136 levels was negatively correlated with the severity of KOA.

As a potent inflammatory factor, IL-17 plays an important role in the development of a variety of inflammatory diseases, and it is also an important regulator in the development of bone metabolism and bone diseases (28). It is suggested that IL-17 can regulate osteoblast differentiation and bone salt deposition. Meanwhile, through the stimulation of osteoblasts, bone cells, and stromal cells, IL-17 can enhance osteoclast activity and promote bone degradation $(12,29)$. Targeting IL-17 or IL-17 receptors (IL-17R) is becoming a hot spot for the treatment of inflammatory diseases and inflammatory bone disease (29,30). Chen et al conducted a retrospective study and reported that Serum IL-17 concentrations were much higher in Han Chinese patients with primary KOA $(n=98)$ than those in healthy control $(n=50)$ (29). Deligne et al explored the expression of IL-17 in the inflamed and the non-inflamed area of each synovium sample $(n=20)$ from human osteoarthritic knee tissues (30). They demonstrated the expression of IL-17 is much higher in the the inflamed than that of the non-inflamed area (30). Both of them pointed out that IL-17 may be effective in the early prevention and therapy for KOA patients $(29,30)$. Therefore, in the future, it will be important to study the signal transduction and pathogenic mechanisms of IL-17/IL-17R in order to find a new therapeutic target for KOA therapy.

In the current study, we also evaluated the level of IL-17 in the serum of KOA patients and healthy controls. Our data showed that the increase in serum IL-17 levels positively correlated with the severity of KOA. The negative correlation between IL-17 and miR-136 promoted us to explore its underlying mechanism. Interestingly, we found that IL-17 is a target gene of miR-136. Further analysis showed that plasma miR-136 levels could be used as a biomarker to screen KOA patients from healthy controls.

In summary, for the first time, we found that plasma miR-136 levels were significantly decreased in KOA patients. More importantly, by targeting IL-17, miR-136 could be used as a potential biomarker for KOA patients.

\section{Acknowledgements}

Not applicable.

\section{Funding}

The present study was supported by a grant from Scientific Research Starting Foundation of Rizhao People's Hospital (grant no. 20160823).

\section{Availability of data and materials}

The datasets used and/or analyzed during the current study are available from the corresponding author on reasonable request.

\section{Authors' contributions}

WL performed the experiments and analyzed the data. ZQ, NG, XT, DC performed part of the RT-qPCR experiments. WS designed the experiments, analyzed the data and gave final approval of the version to be published. All authors read and approved the final manuscript.

\section{Ethics approval and consent to participate}

The present study was approved by the Research Ethics Committee of Rizhao People's Hospital (Rizhao, China) and 
all the patients have provided written informed consent for this study.

\section{Patient consent for publication}

Informed consent for participation in the study or use of their tissue was obtained from all participants.

\section{Competing interests}

The authors declare that they have no competing interests.

\section{References}

1. Evaniew AL and Evaniew N: Knee osteoarthritis: Therapeutic alternatives in primary care. World J Orthop 8: 187-191, 2017.

2. Monfort J, Pujol J, Contreras-Rodriguez O, Llorente-Onaindia J, López-Solà M, Blanco-Hinojo L, Vergés J, Herrero M, Sánchez L, Ortiz H, et al: Effects of chondroitin sulfate on brain response to painful stimulation in knee osteoarthritis patients. A randomized, double-blind, placebo-controlled functional magnetic resonance imaging study. Med Clin (Barc) 148: 539-547, 2017 (In English, Spanish).

3. Niu J, Clancy M, Aliabadi P, Vasan R and Felson DT: Metabolic syndrome, its components, and knee osteoarthritis: The Framingham Osteoarthritis Study. Arthritis Rheumatol 69: 1194-1203, 2017.

4. van Leeuwen DM, van de Bunt F, de Ruiter CJ, van Schoor NM, Deeg DJH and Emanuel KS: Functioning without cartilage: Older people with radiographic knee osteoarthritis who self-report no functional limitations do score lower on a performance battery. J Aging Phys Act 25: 570-575, 2017.

5. Rongen JJ, Rovers MM, van Tienen TG, Buma P and Hannink G: Increased risk for knee replacement surgery after arthroscopic surgery for degenerative meniscal tears: A multi-center longitudinal observational study using data from the osteoarthritis initiative. Osteoarthritis Cartilage 25: 23-29, 2017.

6. Oo WM, Linklater JM and Hunter DJ: Imaging in knee osteoarthritis. Curr Opin Rheumatol 29: 86-95, 2017.

7. Wang K, Xu J, Cai J, Zheng S, Han W, Antony B and Ding C: Serum levels of interleukin-17 and adiponectin are associated with infrapatellar fat pad volume and signal intensity alteration in patients with knee osteoarthritis. Arthritis Res Ther 18: 193, 2016.

8. Wang K, Xu J, Cai J, Zheng S, Yang X and Ding C: Serum levels of resistin and interleukin-17 are associated with increased cartilage defects and bone marrow lesions in patients with knee osteoarthritis. Mod Rheumatol 27: 339-344, 2017.

9. Suurmond J, Dorjée AL, Boon MR, Knol EF, Huizinga TW, Toes RE and Schuerwegh AJ: Mast cells are the main interleukin 17-positive cells in anticitrullinated protein antibody-positive and -negative rheumatoid arthritis and osteoarthritis synovium. Arthritis Res Ther 13: R150, 2011.

10. van Baarsen LG, Lebre MC, van der Coelen D, Aarrass S, Tang MW, Ramwadhdoebe TH, Gerlag DM and Tak PP: Heterogeneous expression pattern of interleukin 17A (IL-17A), IL-17F and their receptors in synovium of rheumatoid arthritis, psoriatic arthritis and osteoarthritis: Possible explanation for nonresponse to anti-IL-17 therapy? Arthritis Res Ther 16: 426, 2014.

11. Southam L, Heath O, Chapman K and Loughlin J: Association analysis of the interleukin 17 genes IL17A and IL17F as potential osteoarthritis susceptibility loci. Ann Rheum Dis 65: 556-557, 2006.

12. Attur MG, Patel RN, Abramson SB and Amin AR: Interleukin-17 up-regulation of nitric oxide production in human osteoarthritis cartilage. Arthritis Rheum 40: 1050-1053, 1997.

13. Honorati MC, Bovara M, Cattini L, Piacentini A and Facchini A: Contribution of interleukin 17 to human cartilage degradation and synovial inflammation in osteoarthritis. Osteoarthritis Cartilage 10: 799-807, 2002.
14. Hu W, Zhang W, Li F, Guo F and Chen A: miR-139 is up-regulated in osteoarthritis and inhibits chondrocyte proliferation and migration possibly via suppressing EIF4G2 and IGF1R. Biochem Biophys Res Commun 474: 296-302, 2016.

15. Lu X, Lin J, Jin J, Qian W and Weng X: Hsa-miR-15a exerts protective effects against osteoarthritis by targeting aggrecanase-2 (ADAMTS5) in human chondrocytes. Int J Mol Med 37: 509-516, 2016.

16. Borgonio Cuadra VM, González-Huerta NC, Romero-Córdoba S, Hidalgo-Miranda A and Miranda-Duarte A: Altered expression of circulating microRNA in plasma of patients with primary osteoarthritis and in silico analysis of their pathways. PLoS One 9: e97690, 2014.

17. Jin R, Xu S, Lin X and Shen M: MiR-136 controls neurocytes apoptosis by regulating Tissue Inhibitor of Metalloproteinases-3 in spinal cord ischemic injury. Biomed Pharmacother 94: 47-54, 2017.

18. Yan M, Li X, Tong D, Han C, Zhao R, He Y and Jin X: miR-136 suppresses tumor invasion and metastasis by targeting RASAL2 in triple-negative breast cancer. Oncol Rep 36: 65-71, 2016.

19. Altman R, Asch E, Bloch D, Bole G, Borenstein D, Brandt K, Christy W, Cooke TD, Greenwald R, Hochberg M, et al: Development of criteria for the classification and reporting of osteoarthritis. Classification of osteoarthritis of the knee. Diagnostic and Therapeutic Criteria Committee of the American Rheumatism Association. Arthritis Rheum 29: 1039-1049, 1986.

20. Kellgren JH and Lawrence JS: Radiological assessment of osteo-arthrosis. Ann Rheum Dis 16: 494-502, 1957.

21. Livak KJ and Schmittgen TD: Analysis of relative gene expression data using real-time quantitative PCR and the 2(-Delta Delta C(T)) method. Methods 25: 402-408, 2001.

22. Pas HI, Winters M, Haisma HJ, Koenis MJ, Tol JL and Moen MH: Stem cell injections in knee osteoarthritis: A systematic review of the literature. Br J Sports Med 51: 1125-1133, 2017.

23. Pihl K, Englund M, Lohmander LS, Jørgensen U, Nissen N, Schjerning J and Thorlund JB: Signs of knee osteoarthritis common in 620 patients undergoing arthroscopic surgery for meniscal tear. Acta Orthop 88: 90-95, 2017.

24. Gu R, Liu N, Luo S, Huang W, Zha Z and Yang J: MicroRNA-9 regulates the development of knee osteoarthritis through the NF-kappaB1 pathway in chondrocytes. Medicine (Baltimore) 95: e4315, 2016.

25. Ko JY, Lee MS, Lian WS, Weng WT, Sun YC, Chen YS and Wang FS: MicroRNA-29a counteracts synovitis in knee osteoarthritis pathogenesis by targeting VEGF. Sci Rep 7: 3584, 2017.

26. He J,Zhao J,Peng X, Shi X, Zong S and Zeng G: Molecular mechanism of MiR-136-5p targeting NF-кB/A20 in the IL-17-mediated inflammatory response after spinal cord injury. Cell Physiol Biochem 44: 1224-1241, 2017.

27. Peng X, Shi X, Zhao J, He J, Li K, Cen Z, Wu Y, Zong S and Zeng G: The effects of miR-136-5p-mediated regulation of A20 in astrocytes from cultured spinal cord cultured cells in vitro. Cell Physiol Biochem 41: 1596-1604, 2017.

28. Retraction note: Mast cells are the main interleukin 17-positive cells in anticitrullinated protein antibody-positive and -negative rheumatoid arthritis and osteoarthritis synovium. Arthritis Res Ther 17: 354, 2015.

29. Chen B, Deng Y, Tan Y, Qin J and Chen LB: Association between severity of knee osteoarthritis and serum and synovial fluid interleukin 17 concentrations. J Int Med Res 42: 138-144, 2014.

30. Deligne C, Casulli S, Pigenet A, Bougault C, Campillo-Gimenez L, Nourissat G, Berenbaum F, Elbim C and Houard X: Differential expression of interleukin-17 and interleukin-22 in inflamed and non-inflamed synovium from osteoarthritis patients. Osteoarthritis Cartilage 23: 1843-1852, 2015.

This work is licensed under a Creative Commons Attribution-NonCommercial-NoDerivatives 4.0 International (CC BY-NC-ND 4.0) License. 